\title{
The Tiered Pharmacy Benefit: Challenges in Today's Health Care Market
}

\author{
James T. Kenney, RPh, MBA
}

$\mathrm{W}$ Then I started my career in managed care in 1980, the pharmacy benefit was an add-on to the standard medical coverage and not viewed as a particularly valuable benefit by employer groups. A separate rider was offered to employers to add this coverage, and many balked at the additional modest cost to expand their benefits to include drugs. One third of the membership had this benefit in those early years, with a 2-tier generic-brand design and copays of $\$ 1$ and $\$ 3$ for up to a 30-day supply. Some plans even offered up to a 6-month supply at a single dispensing and chose to market the pharmacy benefit as a point of differentiation from competitors. The uptake of this benefit option was significant over the next 10 years because patients wanted insurance coverage for medications and predictable out-of-pocket costs. By the end of the decade, nearly 100\% of members had a drug benefit, and employers required this as part of the standard benefit package for their employees.

Published in 2003, the accompanying JMCP article "Effects of a 3-Tier Pharmacy Benefit Design on the Prescription Purchasing Behavior of Individuals with Chronic Disease" examines the shift from 2-tier to 3-tier designs 10 years later, with a focus on achieving 2 primary objectives: (1) to promote an increase in the use of lower-cost generic products and (2) to increase the use of formulary agents while reducing the use of nonformulary products. The results of the analysis supported these objectives and also raised concerns regarding the need for patients with chronic diseases to have access to appropriate medications at reasonable out-of-pocket costs.

The pharmacy benefit has come a long way since these early versions when drug costs were very low, many diseases could be treated with branded and generic drugs from different therapeutic classes, and the categories of orphan disease treatments or specialty drugs were essentially nonexistent. These early pioneers of pharmacy benefit management shared the same clinical, professional, financial, and patient-focused concerns of pharmacy managers in the health care market today.

J Manag Care Spec Pharm. 2020;26(5):586-87

Copyright $\odot 2020$, Academy of Managed Care Pharmacy. All rights reserved.
Rebates were not mentioned in the 2003 article as a key driver for the formulary tiering, although they were used in the market at that time. The total volume and dollar values of the rebate programs were not significant enough to aggressively drive formulary decisions. This has certainly changed in the market today, with rebates playing a significant role in the drug review and coverage process. In many cases, rebates may be a principle driver for formulary positioning in competitive therapeutic classes. Formulary managers must balance the clinical, qualitative, and financial components of each medication to achieve an optimal benefit design that meets the needs of patients, providers, and payers.

As referenced in the JMCP article, the challenges of addressing double-digit cost increases while maintaining choice for providers and patients is practiced today, as well. While adherence was identified as a concern, the article focused more on the potential for reduced adherence due to increased patient cost or restrictions due to the nonformulary positioning of products. Today, we continue to be concerned about adherence; however, additional factors are considered because of the scientific complexity and toxicities of many agents in the market today. The nonadherence issue continues to plague our system, with rates hovering around 50\% for many chronic diseases.

We are all well aware of the typical benefit designs employed today in the market, with 4-tier and 5-tier closed formulary designs as the most prevalent. Patients are often required to pay full price for nonformulary or noncovered products. The significant number of approvals by the U.S. Food and Drug Administration in recent years, including many orphan drugs and other high-cost specialty drugs, has led to an explosion of unique tier designs and structures to blunt the cost of these agents. Copays are being replaced by coinsurance, and many patients must satisfy deductibles for pharmacy and medical expenses before more broad coverage and reduced out-ofpocket benefits are available.

In the market today, we continue to see significant use of generic drugs at rates of $85 \%-90 \%$ of total prescription volume across all patient groups, including commercial, Medicare, Medicaid, and the health exchanges. The bigger challenge that 
health plans face today is the management of high-cost specialty medications, and traditional formulary tier designs that do not provide the adequate methodology to control the cost of these agents. Coinsurance is used for most of these agents, although, there is usually a cap on the total out-of-pocket cost per prescription to help the patients afford these medications.

Employers continue to pressure health plans to lower their monthly premiums for the fully insured business or the administrative fees for the self-insured business. These demands have led to an increase in the use of deductibles for pharmacy and medical benefits. These deductibles can be $\$ 2,000-\$ 5,000$ per year for an individual or family plan, with most patients experiencing these costs for medication use. Virtually all specialty medications would require the patients to spend $\$ 2,000$ $\$ 5,000$ for the first prescription of the benefit year before traditional benefits would apply. Plans continue to struggle with balancing the need to lower costs and allow reasonable access to products for patients with chronic or rare diseases.

A majority of manufacturers now offer copay assistance programs for select medications, and patients can access these to offset the cost of the medications at the pharmacy. In many cases, this assistance can apply to the deductibles and help the patients satisfy this requirement and gain access to more standard copay or coinsurance coverage for their medications. Recently, a number of plans have begun to employ copayaccumulator programs that do not allow manufacturer assistance to satisfy deductible requirements for the pharmacy benefit. This would be an interesting area of future study to examine the effect of these programs on patient compliance and persistence with drug therapy or the potential use of the emergency room as a lower cost option due to standard copays that may be lower than the patient's pharmacy deductible limits.

Another significant market change has been a focus on shifting from volume to value in how we manage benefits and medications. Many plans have now turned their attention to value-based insurance designs and outcomes-based pharmaceutical contracting. For many years, the health plan formulary committees would evaluate new drugs and make coverage decisions based on the pivotal clinical trial data and rarely track or monitor actual outcomes going forward. Today, we see many plans requiring manufacturers to go at risk for the cost of medications when expected clinical outcomes are not achieved. This is a particular need as we see an expansion of multimillion-dollar cell and gene therapies that may provide long-term benefit provided that patients respond to treatment.

We now face unprecedented changes in our health care system with the explosion of new treatments and technologies. New pioneers are needed to develop innovative and comprehensive strategies to meet this demand. AMCP continues to provide the tools, resources, and forum to help drive this process as we face a future with endless possibilities to discover and unlock the keys to successful management and treatment of chronic and rare diseases in our patient populations.

\section{Authors}

JAMES T. KENNEY, RPh, MBA, President, JTKENNEY, LLC, Waltham, Massachusetts, and President of the AMCP Board of Directors, Alexandria, Virginia.

AUTHOR CORRESPONDENCE: James T. Kenney, RPh, MBA, can be contacted at jtkenneyllı@comcast.net.

\section{DISCLOSURES}

No funding supported the writing of this reflection. The author has nothing to disclose. 